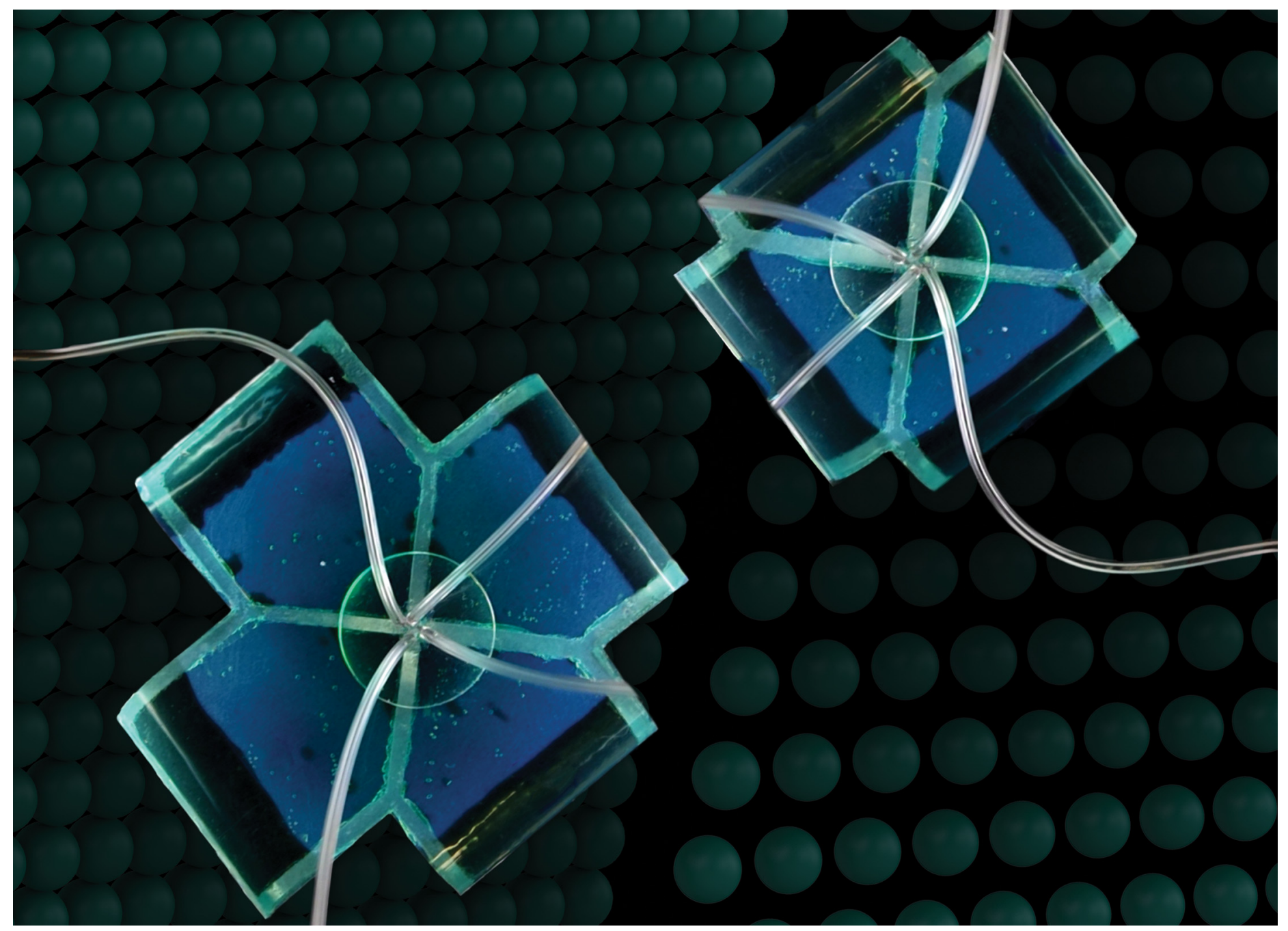

Showcasing research from Prof. Michael D. Bartlett's Soft Materials and Structures Lab, Mechanical Engineering, Virginia Tech.

Deterministic control of adhesive crack propagation through jamming based switchable adhesives

Adhesive crack dynamics are controlled by integrating a rigidity tuning granular jamming layer into adhesive films. This highly reversible mechanism can initiate, propagate, and arrest adhesive cracks at arbitrary selectable locations in multiple peel directions.

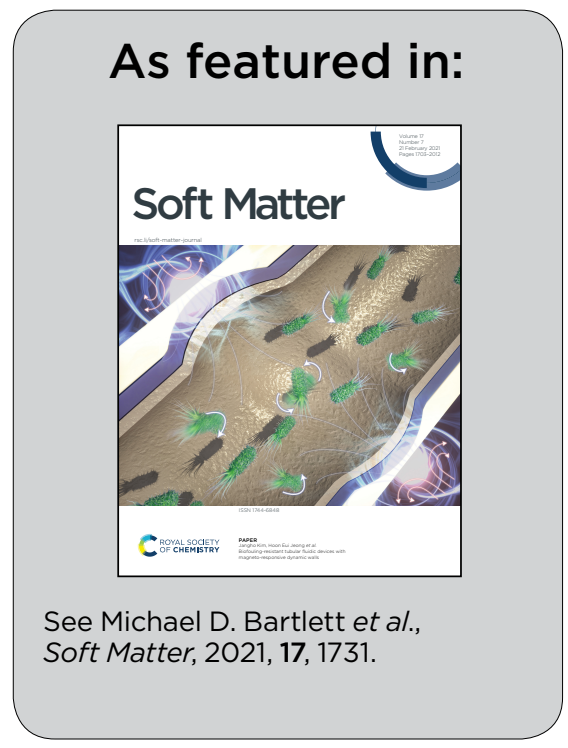

rsc.li/soft-matter-journal 
Check for updates

Cite this: Soft Matter, 2021,

17, 1731

Received 30th November 2020,

Accepted 15th January 2021

DOI: $10.1039 / \mathrm{d} 0 \mathrm{sm} 02129 f$

rsc.li/soft-matter-journal

\section{Deterministic control of adhesive crack propagation through jamming based switchable adhesives $\uparrow$}

\author{
Cole B. Haverkamp, $\ddagger^{\mathrm{a}}$ Dohgyu Hwang, $\ddagger^{\mathrm{bc}}$ Chanhong Lee ${ }^{\mathrm{a}}$ and \\ Michael D. Bartlett (iD $* b c$
}

\begin{abstract}
Controlling delamination across a material interface is a foundation of adhesive science and technology. This ranges from creating permanent, strong adhesives which limit crack propagation to reversible adhesives which initiate cracks for release. Methods which dynamically control cracks can lead to more robust adhesion, however specific control of crack initiation, propagation, and arresting is challenging because time scales of crack propagation are much faster than times scales of mechanisms to arrest cracks. Here we show the deterministic control of crack initiation, propagation, and arresting by integrating a granular jamming layer into adhesive films. This allows for controlled initiation of a propagating crack by reducing rigidity and then rapidly arresting the crack through jamming, with a rise in stiffness and an $11 \times$ enhancement in adhesion. This process is highly reversible and programmable, allowing for numerous crack initiation, propagation, and arresting cycles at arbitrary selectable locations in a peeling adhesive. We demonstrate this crack-control approach in single and multiple peel directions under fixed load conditions in response to diverse pressurization input signal profiles (i.e. time varying propagation and arresting scenarios).
\end{abstract}

\section{Introduction}

Controlling adhesive bonding and delamination is essential to diverse applications in consumer and healthcare products, robotics, locomotion, and construction. ${ }^{1-7}$ Adhesive bonding is governed by a combination of interfacial properties, contact geometry, and mechanical properties which can be varied to create diverse adhesives. These range from permanent, strong adhesives which limit crack propagation to reversible adhesives which initiate cracks for release. Therefore, determining how a crack initiates, propagates, and arrests is essential to control the strength, toughness, and reversibility of adhesive interfaces. ${ }^{8-10}$ This is often evaluated through a fracture mechanics energy balance, where cracks begin to propagate when the strain energy release rate reaches a defined critical strain energy release rate. ${ }^{11,12}$ Under the conditions of constant load, once a crack

\footnotetext{
${ }^{a}$ Department of Materials Science \& Engineering, Iowa State University, Ames, IA 50011, USA

${ }^{b}$ Macromolecules Innovation Institute, Virginia Tech, Blacksburg, VA 24061, USA. E-mail: mbartlett@vt.edu

${ }^{c}$ Department of Mechanical Engineering, Soft Materials and Structures Lab,

Virginia Tech, Blacksburg, VA 24061, USA

$\dagger$ Electronic supplementary information (ESI) available. See DOI: 10.1039/ d0sm02129f

\$ These authors contributed equally to this work.
}

begins to propagate, the interface commonly separates completely. Specifically controlling how cracks move can be challenging. For example, adhesives commonly focus on increasing dissipation at the interface as a mechanism to increase adhesion strength. ${ }^{13}$ This leads to an intrinsic trade-off as interfacial dissipation tends to lead to high forces for both crack initiation and propagation, reducing the ability to decouple crack dynamics for controllable bonding and release.

The dynamic control of crack behavior is key in emerging technologies, such as wearable electronics, robotics, and transfer printing that require strong adhesion during use but need to be released upon completion of their function. ${ }^{14-18}$ This requires switchable adhesives, where the adhesive force can be modulated between a high and low adhesive state. ${ }^{19}$ Switchable adhesives explicitly control the initiation and propagation of interfacial cracks. By raising the force required to initiate cracks while maintaining low force during propagation, adhesives can be switched from a high to low adhesive state. ${ }^{20,21} \mathrm{~A}$ promising approach to create switchable adhesives is through the dynamic control of interfacial structure and rigidity. One of the primary methods to control rigidity has been through thermally induced phase change. Here, a material can be dramatically softened by heating above a transition temperature, inducing a stiffness change from a rigid to a compliant material, resulting in control of adhesion strength. ${ }^{2-25}$ However, thermally induced phase 
change is limited by heat transfer in soft materials, which often results in slow rigidity switching. ${ }^{26,27}$ This results in challenges in controlling cracks, as the time scale of crack propagation is much faster than the times scale for mechanisms which arrest cracks.

Pneumatically activated systems can be rapid and have been utilized to tune surface structure for adhesion control. ${ }^{28-31}$ Pneumatic systems can also control contact geometry and stiffness which can lead to rapidly switchable adhesives. ${ }^{32}$ Particle jamming under reduced pressure is another approach which can rapidly transition granular media from a flowable fluid to a rigid solid, ${ }^{33-36}$ enabling rigidity to be dynamically switched and programmed. This has been demonstrated in robotic grippers which can increase in rigidity to grasp objects and then soften to release. ${ }^{37,38}$ However, jamming mechanisms have primarily relied on mechanical interlocking to pick up and manipulated objects, and determining how interfacial jamming manipulates adhesion and adhesive crack dynamics presents opportunities to develop and understand rapidly switchable interfaces.

Here we show the deterministic control of adhesive crack dynamics including initiation, propagation, and arrest by integrating a rigidity tuning granular jamming layer into an adhesive film (Fig. 1a and Movie S1, ESI $\dagger$ ). By controlling the pneumatic pressure in a granular filled cavity, rigidity is rapidly tuned to propagate and arrest cracks. By applying negative pressure to the cavity, granular jamming occurs and causes an increase in the stiffness of the interface, which results in an increased adhesive force by a factor of $\sim 11 \times$ and crack arrest. Upon removal of the pneumatic pressure, the interface rapidly softens, and the adhesive force required to separate the interface dramatically decreases. This interface softening results in crack propagation. This process is highly reversible and programmable, allowing for numerous crack initiation, propagation, and arresting cycles at arbitrary selectable locations in a peeling adhesive. Jamming layers can be deployed as patterns or as single continuous segments, allowing for adhesion control in pre-determined locations defined by patterns or at dynamically selectable locations in continuous jamming segments. These behaviors are demonstrated by hanging a weight on an adhesive strip and monitoring the crack position as a function of time (see Fig. 1b). When unjammed, cracks propagate, and when jammed, the cracks arrest as indicated by the constant crack position. We find that each crack propagation and arrest event is rapidly activated upon switching of the granular jamming state, which offers a new mechanism for dynamic, rapid control of crack behaviors.

\section{Results and discussion}

Adhesive design for deterministic control of adhesion

The jamming-based switchable adhesive consists of a rigidity tunable granular jamming layer, a polyethylene terephthalate
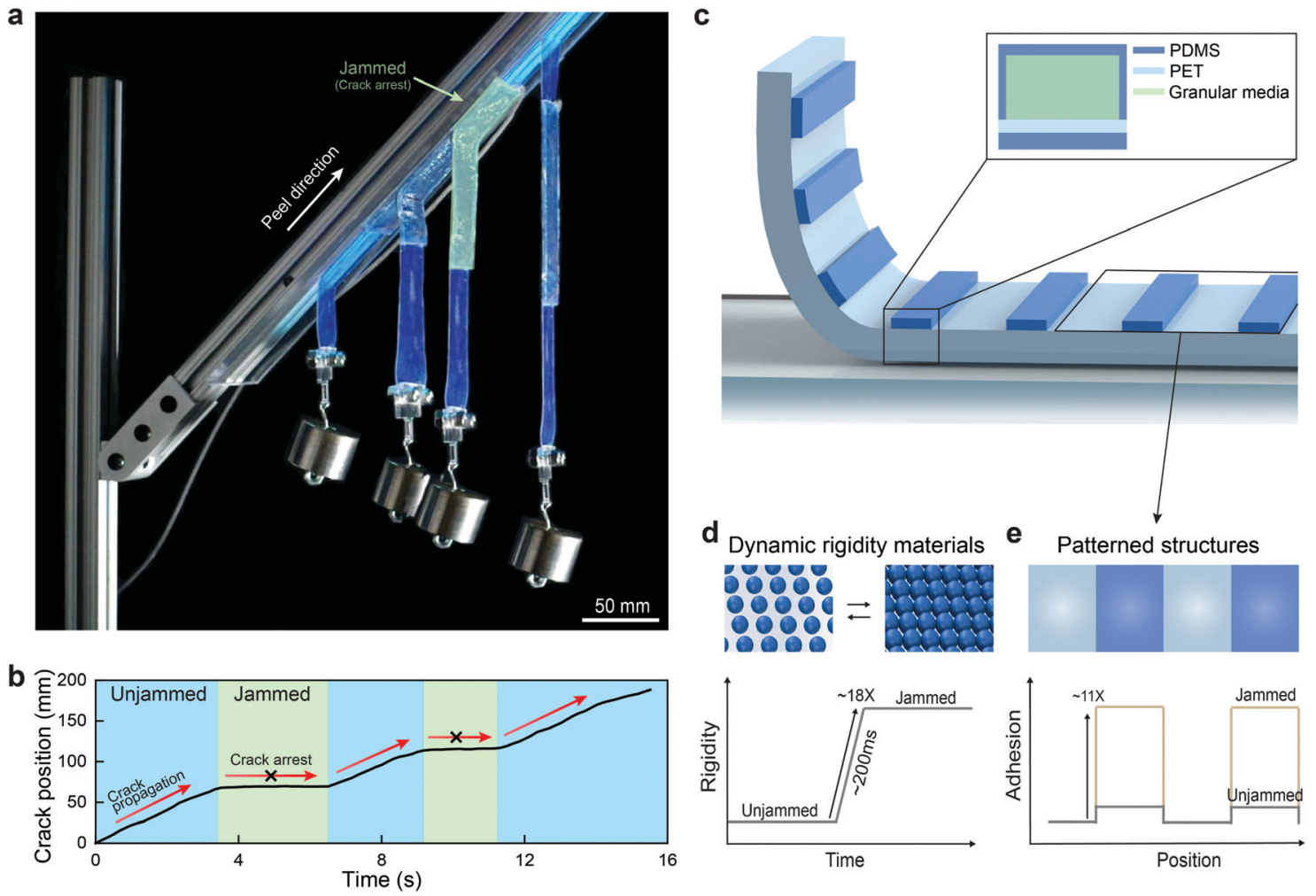

Fig. 1 Deterministic control of adhesion crack propagation. (a) Image sequence showing the jamming based switchable adhesive for deterministic control of adhesive crack propagation (Movie S1, ESI $\dagger$ ). (b) Crack position versus time for dynamic crack propagation with regions indicating unjammed and jammed states. (c) Schematics of the jamming-based switchable adhesive. (d) Rigidity can be rapidly tuned by a factor of $\sim 18$ within $\sim 200 \mathrm{~ms}$. (e) The rigidity-tunable region shows an increase in adhesion by a factor of $\sim 11$ when jammed, relative to the unpatterned region. 
(PET) backing layer, and a polydimethylsiloxane (PDMS) adhesive layer. The granular jamming layer is bonded on the backing layer and consists of ground coffee particles encapsulated by a PDMS membrane (Fig. 1c). The jamming layers are connected to a pneumatic system for controlling the inner pressure. When the jamming layers are relaxed at ambient pressure, the granular particles freely move during the bending of the adhesive strip under peeling (Fig. 1d). Thus, the bending rigidity is predominantly determined by the bi-layered adhesive strip. However, when applying a negative pressure, the granular particles jam which rapidly restricts deformation within $\sim 200 \mathrm{~ms}$. Tight packing of granular particles increases the bending rigidity by a factor of $\sim 18$ relative to the ambient pressure condition. The jamming layer can be continuous or patterned across the backing layer. This allows for modulation of stiffness spatially through a time varying pressure in continuous layers, or constant jamming pressure in patterned layers where spatial stiffness control is determined by the spacing of the jamming layer patterns on the backing layer (Fig. 1e). The jamming layerintegrated regions allow for dynamic adhesion control by a factor of $\sim 11$, relative to the homogeneous region which shows a static, steady-state adhesion force.

\section{Properties of jamming-based switchable adhesives}

We perform a set of experiments to determine the mechanical properties of the jamming-based switchable adhesives. We first conduct a three-point flexural test on the jamming layer under various jamming pressures $(\Delta P=0,-30,-60$, and $-87 \mathrm{kPa})$ to analyze the bending rigidity of the adhesive (Fig. 2a). The jamming layer under a negative pressure $(\Delta P=-87 \mathrm{kPa})$ shows a rigid response with a bending rigidity of $870 \pm 25 \mathrm{~N} \mathrm{~m}^{-1}$, and the mechanical response can be further tuned by controlling the pneumatic pressure $\left(630 \pm 70 \mathrm{~N} \mathrm{~m}^{-1}\right.$ and $470 \pm 20 \mathrm{~N} \mathrm{~m}^{-1}$ at $\Delta P=-60$ and $-30 \mathrm{kPa}$, respectively). In contrast, the jamming layer under an ambient pressure $(\Delta P=0 \mathrm{kPa})$ exhibits a soft response with a bending rigidity of $50 \pm 2 \mathrm{~N} \mathrm{~m}^{-1}$, which is $\sim 18$ times softer than the pneumatically pressured state $(\Delta P=-87 \mathrm{kPa})$.

To enable an instantaneous arrest and reinitiation of crack propagation, the rigidity-tunable jamming layer is controlled through a pneumatic system. Fig. $2 b$ shows a representative pressure curve as a function of time, where a prescribed jammed state is attained within $\sim 200 \mathrm{~ms}$ and reduced to an unjammed state within $\sim 50 \mathrm{~ms}$, which rapidly controls the rigidity of jamming layers and the crack propagation. Further, the jamming pressure can be held constant to systematically evaluate the adhesive response as a function of jamming pressure.

To determine adhesion tunability in response to jamming, we perform a 90 degree peel test on the jamming-based adhesive under various pressures $(\Delta P=0,-30,-60$, and $-87 \mathrm{kPa})$ (Fig. $2 \mathrm{c}$ and $\mathrm{d}$ ). At the onset of peeling (displacement $=0-6 \mathrm{~mm}$ in Fig. 2d), the crack propagates in the jamming layer-free region at
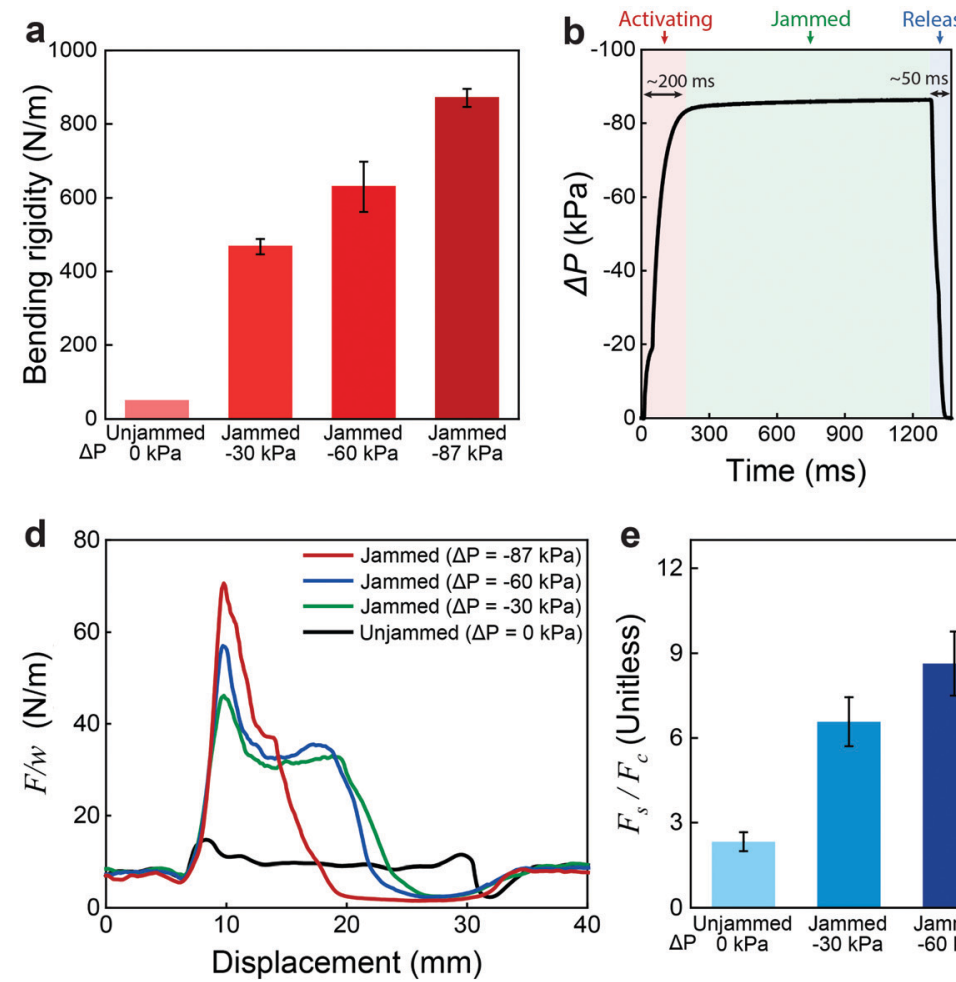

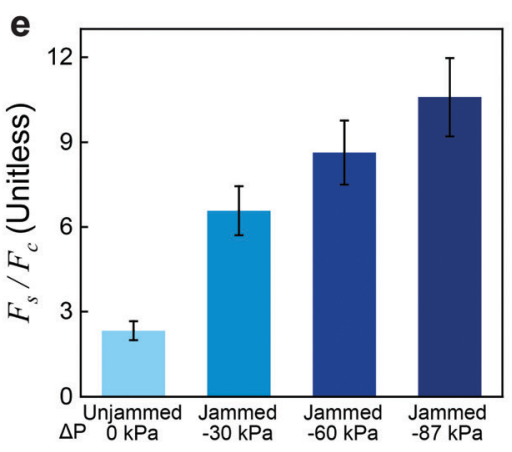

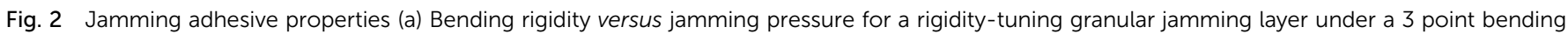

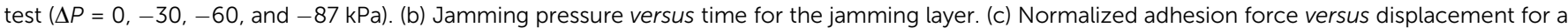

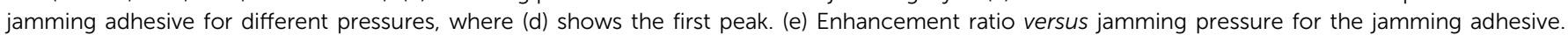
(f) Peak adhesion force in the stiff region versus bending rigidity. The dashed line denotes the linear relation between the two properties. 
a constant rate which is equal to the machine displacement rate $\left(=1 \mathrm{~mm} \mathrm{~s}^{-1}\right)$. The peel region shows a steady-state normalized peel force with $F / w=G_{\mathrm{c}}$ of $6.8 \pm 0.8 \mathrm{~N} \mathrm{~m}^{-1}$, where $w$ is the width of the strip and $G_{\mathrm{c}}$ is the critical energy release rate for interfacial failure. ${ }^{12}$ However, when the crack meets a jamming layer interface, it is arrested due to an increased bending rigidity through jamming. In Fig. 2c, each peak corresponds to an individual jamming layer segment. This segment raises the peak, normalized adhesion forces to $45 \pm 2 \mathrm{~N} \mathrm{~m}^{-1}, 59 \pm 3 \mathrm{~N} \mathrm{~m}^{-1}$, and $72 \pm 3 \mathrm{~N} \mathrm{~m}^{-1}$ for $\Delta P=-30,-60$, and $-87 \mathrm{kPa}$, respectively. After the peak force, an intermediate plateau peel force is observed as the crack moves through the jamming layer. This force is larger than the steady state force observed in the compliant region and is most noticeable at intermediate jamming pressures. We note that this plateau is not typically observed in solid stiff regions, ${ }^{39,40}$ and points to a unique behavior of the jammed granular media for adhesion control. Lastly, as the crack finishes propagating through the stiff region and reaches the compliant interface, the peel force decreases and then reaches a steady state force in the jamming layer-free region.

The relationship between adhesion force and bending rigidity for an adhesive with a constant width can be described as: $F_{\mathrm{s}} / F_{\mathrm{c}}$ $\sim E_{\mathrm{s}} I_{\mathrm{s}} / E_{\mathrm{c}} I_{\mathrm{c}}$, where the subscripts s and $\mathrm{c}$ indicate the stiff and the compliant region, respectively. ${ }^{39-43}$ The stiff/compliant interface alters the shape of the bent adhesive strip, which modifies the mechanical energy in the adhesion system. The change in shape of the bent strip depends upon the bending rigidity $E I$ of the two regions, in which $E$ is the modulus and $I$ is the second moment of area. The equation shows that the adhesion force can be enhanced by increasing the bending rigidity of a stiff region $E_{\mathrm{S}} I_{\mathrm{S}}$ relative to that of a compliant region $E_{\mathrm{c}} I_{\mathrm{c}}$.

In our system, the stiff segment corresponds to the region with pneumatically controlled jamming layers. Fig. 2e shows the adhesion enhancement ratio $\left(F_{\mathrm{s}} / F_{\mathrm{c}}\right)$ of the jamming-based switchable adhesive for four different pressures (here $F_{\mathrm{c}}$ is adhesive force in the unpatterned region without a jamming layer). The largest enhancement ratio $(\sim 11)$ is achieved when a high negative pressure $(\Delta P=-87 \mathrm{kPa})$ is applied, while the smallest is achieved $(\sim 2)$ under an ambient pressure $(\Delta P=0 \mathrm{kPa})$. The slight adhesion enhancement for the unjammed state originates from the finite thickness of the elastomeric walls that encapsulate the granular particles in the jamming layer. We note that the $F_{\mathrm{S}} / F_{\mathrm{c}}$ enhancement at maximum jamming pressure is lower than expected based on the $E_{\mathrm{S}} I_{\mathrm{S}} / E_{\mathrm{c}} I_{\mathrm{c}}$ ratio. We attribute this discrepancy to the elastomeric edge of the jamming pocket, a non-ideal distribution of particles that creates non-uniform cross-sections of the jamming layer, or inefficient stress-transfer effects from the granular media. These effects have the potential to make the local rigidity contrast more diffuse, reducing the validity of the assumption of an ideally sharp stiff/compliant interface. To analyze the adhesion enhancement without an ideally sharp stiff/compliant interface, we study the correlation between the normalized adhesive force $\left(F_{\mathrm{s}} / w\right)$ and the measured bending rigidity in Fig. 2f. For the samples tested, the adhesion force increases as bending stiffness increases, as indicated by the linear relationship between the two properties. Therefore, even though the adhesion enhancement relative to the unpatterned/ compliant region stiffness $\left(E_{\mathrm{S}} I_{\mathrm{S}} / E_{\mathrm{c}} I_{\mathrm{c}}\right.$ ratio) may be reduced by a
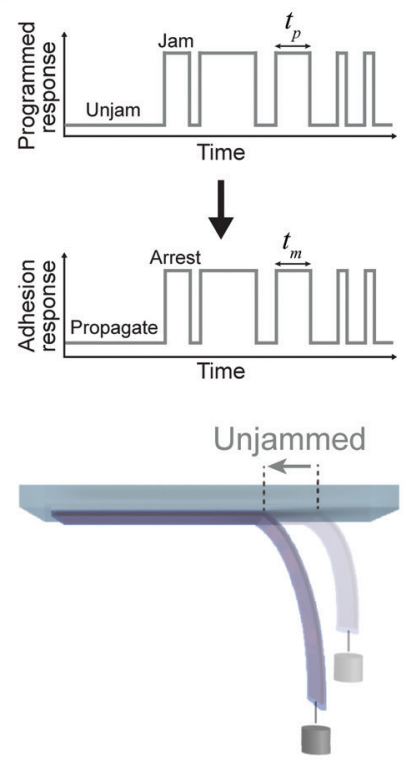

b
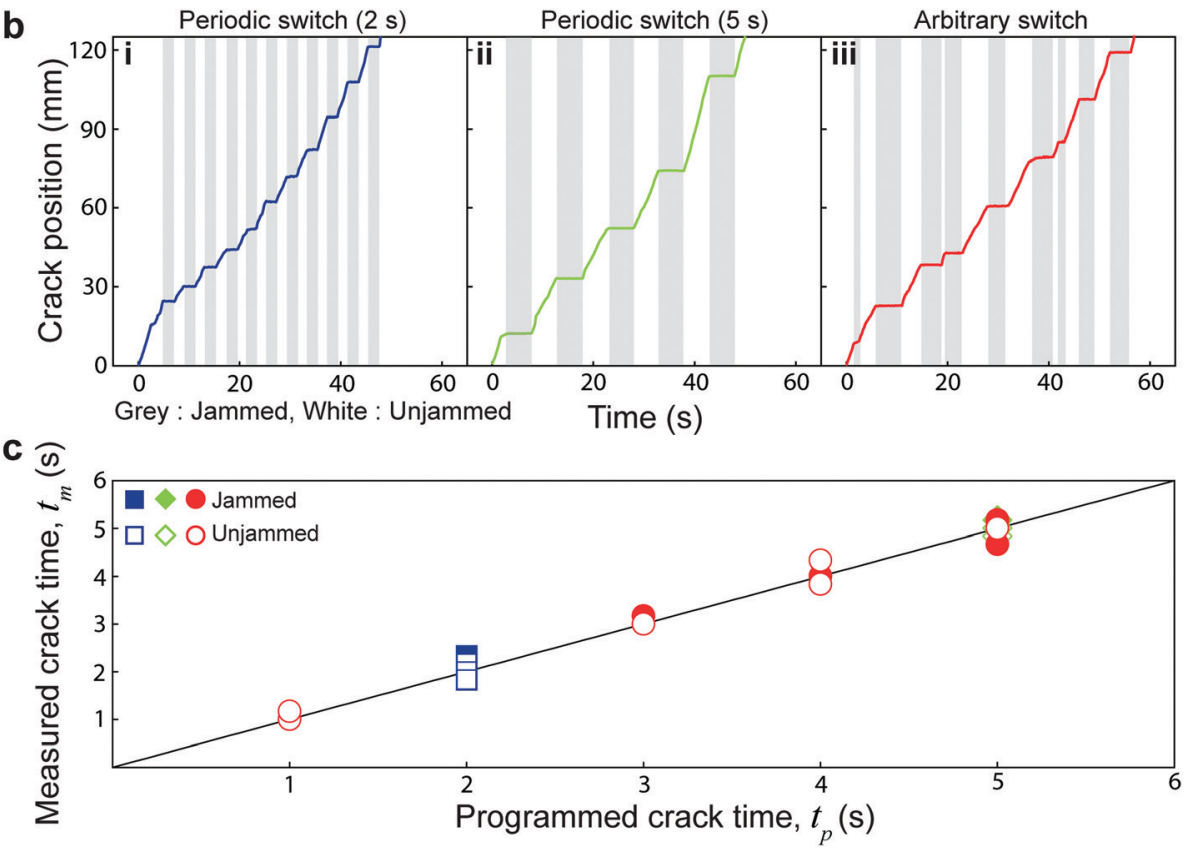

Fig. 3 Programmability of jamming-based switchable adhesion (a) Demonstration of programmable adhesion control. (b) Crack position versus time for three different adhesion control. Cracks are programmed to get arrested in both arbitrary and periodic manners. Grey regions indicate the programmed crack behavior (Movies S2-S4, ESI†). (c) Measured crack propagation (unjammed) and arrest (jammed) show an excellent agreement with the programmed crack behavior. 
a

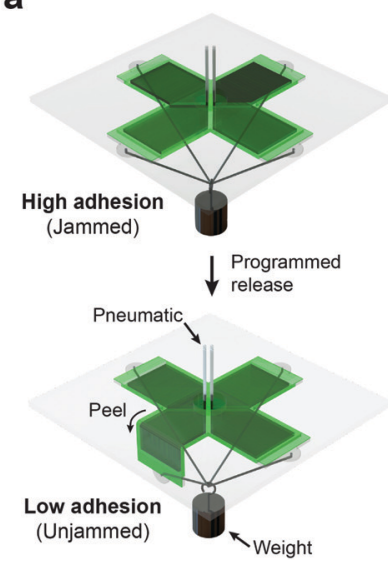

b

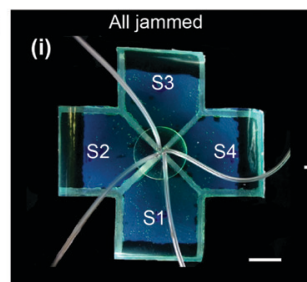

(iv)

Fig. 4 Multi-directional adhesive crack control (a) Schematics of an adhesive with four jammed layer segments (top) and with three jammed segment and an unjammed segment (bottom). (b) Photographs of the independently-controllable adhesive crack propagation in multiple directions. The programmed sequence is: (i) all segments jammed. (ii) S1 and S2 unjammed, S3 and S4 jammed. (iii) S3 and S4 unjammed, S1 and S2 jammed. (iv) all segments are unjammed. Scale $=20 \mathrm{~mm}$ (Movie S5, ESI $\dagger$ ) (c) Relative crack position versus time for four segments during the programmed sequence.

the diffuse stiff/compliant interface, the relative increase in adhesion force once jamming is introduced increases proportionally. This is supported by the three jammed samples (the highest bending rigidity data points) in Fig. 2f, which all fall on the linear line. This demonstrates that dynamically controlling local bending rigidity through particle jamming is an effective approach to tune adhesion force during peeling.

\section{Deterministic crack control}

The rapid rigidity tuning in jamming based switchable adhesives opens opportunities to deterministically control adhesive crack dynamics. This is achieved by controlling initiation of a propagating crack front by reducing rigidity and then rapidly jamming and arresting the crack through a rise in stiffness. We perform experiments where the adhesive consists of a single jamming layer which covers the entire strip, and a hanging weight at the peel end. The single, continuous jamming strip allows for control of cracks at arbitrary selectable locations or times in a peeling adhesive. An output signal from a microcontroller opens and closes a solenoid valve at programmed intervals $\left(t_{\mathrm{p}}\right)$ to jam and unjam the granular media. This results in a corresponding adhesive response where the cracks arrest or propagate for a measured time interval $\left(t_{\mathrm{m}}\right)$. We perform three different sequences of jam-unjam states by varying $t_{\mathrm{p}}$ (Fig. 3a and Movies $\mathrm{S} 2-\mathrm{S} 4$, ESI $\dagger$ ). Fig. 3b presents the crack position as a function of time to show the relationship between the input signal of pressurization and the crack displacement profile. Here the grey regions indicate jamming where the crack position is constant (i.e. arrested) and the white regions are unjammed where the crack is moving (i.e. propagating). In sequence (i) and (ii), the jam-unjam states are switched at every 2 and 5 s, respectively, while in the sequence (iii) switching frequency is randomly generated in $1 \mathrm{~s}$ intervals. In all cases, a sharp transition in the crack dynamics between the propagating and arresting cracks is observed, and the transition occurs rapidly upon switching of the input signal. By plotting the measured crack time $\left(t_{\mathrm{m}}\right)$ as a function of the programmed crack time $\left(t_{\mathrm{p}}\right)$, we find excellent agreement between the measured and programmed crack times in both the jammed and unjammed states over various control times (Fig. 3c). This result demonstrates that the jamming-based rigidity tuning mechanism allows for the ability to deterministically control adhesive crack dynamics on demand.

\section{Crack control in multiple directions}

More complex adhesive designs, such as an array of multiple rigidity-tunable segments can enable discretized adhesion control in multiple directions. We create a cross-shaped adhesive where each arm has a jamming-based rigidity-tunable segment (Fig. 4a and Movie S5, ESI $\dagger$ ). The rigidity of each arm is independently controlled through separate pneumatic controls. Fig. 4b shows the peel of each pneumatically controlled rigiditytunable segment ( $\mathrm{S} 1$ through $\mathrm{S} 4$ ) which is connected to a single weight. In the sequence (ii), two of the four segments (S1 and S2) are unjammed to allow the crack to propagate across the interface, while the other two segments (S3 and S4) remain jammed to prohibit crack propagation. In the next sequence (iii), the two jammed segments (S3 and S4) are released, while the two unjammed segments are now jammed to halt the crack propagation. In the last sequence (iv), all four segments are unjammed, which propagates cracks across in all peel directions for complete removal. The crack propagation as a function of time is characterized in Fig. 4c, which shows that each segment functions independently for controlling the crack movement. This result indicates that the global performance of an adhesive can be fine-tuned through a collection of multiple adhesive segments capable of deterministic control of crack movement, which can be useful for effective load sharing across the interface.

\section{Conclusions}

In this work, we show deterministic control of adhesion crack movement through a jamming-based switchable adhesive. The 
capability to control cracks and adhesion is achieved by dynamically controlling rigidity through an integrated jamming layer. By reducing rigidity, cracks propagate, by rapidly increasing rigidity through jamming, cracks arrest. As the pneumatic system allows for rapid jamming and unjamming, the cracks can arrest at time scales that are faster than the adhesive crack propagation, allowing for new levels of control of adhesive crack dynamics. The granular jamming also enhances adhesion, which is $\sim 11$ times higher than the homogeneous region. By further increasing rigidity contrast between the compliant and stiff segments further enhancement could be obtained, which may be possible by improving encapsulation approaches. Crack dynamics are characterized by applying programmed input signals of pneumatic pressure, demonstrating an in situ control of crack movement and adhesion in both single and multiple peel directions. While this work uses granular jamming, we envision that other strategies such as layer jamming can be used to control adhesion. Also, we expect this mechanism to work in adhesive systems beyond PDMS, however, for much stronger adhesives, other failure mechanisms could emerge which may be related to jamming forces/strength as a limit to tune adhesion. This could arise when the magnitude of rigidity tuning becomes similar to or less than the adhesion fracture energy contributions (i.e. $G_{\mathrm{c}}$ ) to adhesive force capacity. We anticipate that this jamming strategy for rapidly controlling adhesive crack propagation could enable new techniques for adhesion control in soft robotics, wall-climbing apparatus, and pick-and-place applications while providing a tunable system for studying crack dynamics in adhesives.

\section{Experimental}

\section{Jamming adhesive fabrication}

A $\sim 350 \mu \mathrm{m}$ thick layer of PDMS (Sylgard 184 with a 30:1 base : curing agent ratio; Dow Corning) is formed on a glass plate using a thin film applicator (ZUA 2000; Zehntner Testing Instruments), which is then cured in an oven at $80{ }^{\circ} \mathrm{C}$ for $1 \mathrm{~h}$. Subsequently, a PET film (Grainger, $t=25 \mu \mathrm{m} \mathrm{PET}$ ) is treated with oxygen plasma to enhance adhesive bonding with PDMS (30 s, 0.7 torr oxygen at medium RF level, PDC-001-HP, Harrick Plasma). The treated PET film is attached onto the polymer layer, which is then cured in an oven at $80{ }^{\circ} \mathrm{C}$ for at least $20 \mathrm{~h}$. A jamming layer component of PDMS (40:1 base : curing agent ratio), which consists of a top compliant membrane $(t \sim 350 \mu \mathrm{m})$ and side support walls, is created from a replica mold and attached on the PET film surface using glue (Sil-Poxy; SmoothOn). Ground coffee particles are poured into the jamming layer through an unsecured side and are sealed using Sil-Poxy. One side of the jamming layer is punctured using a needle and is connected to the pneumatic system and subsequently sealed with Sil-Poxy.

\section{Three point bending test}

Three point bending tests are performed on a jamming layer with a bi-layered adhesive strip at a displacement rate of $100 \mu \mathrm{m} \mathrm{s}^{-1}$ under four different pressures $(\Delta P=0,-30,-60$, and $-87 \mathrm{kPa})$. The distance between supports is set to be $32 \mathrm{~mm}$.

\section{Adhesion characterization}

The jamming adhesive is tested using a mechanical testing machine (Instron 5944) at a displacement rate of $1 \mathrm{~mm} \mathrm{~s}^{-1}$ using an adhesion test apparatus under a 90 degree peel loading. Between each test, the adhesive surface is cleaned with Isopropyl alcohol (IPA). The adhesive is then adhered onto the test apparatus by using a rubber roller to ensure intimate contact between the adhesive and a substrate of the apparatus. Crack position is measured using Tracker Video Analysis Software. For the single direction crack control the hanging mass is $30 \mathrm{~g}$, for the multi-direction it is $20 \mathrm{~g}$. The pneumatic pressure is controlled using an Arduino Mega with $12 \mathrm{~V}$ solenoid valves (Spartan Scientific), a pressure sensor, and a vacuum reservoir with a pin valve to modulate vacuum pressure.

\section{Conflicts of interest}

There are no conflicts to declare.

\section{Acknowledgements}

The authors acknowledge support through a 3M Non-Tenured Faculty Award.

\section{References}

1 A. Kinloch, Adhesion and Adhesives Science and Technology, Springer, 1987.

2 D. Vella, J. Bico, A. Boudaoud, B. Roman and P. M. Reis, Proc. Natl. Acad. Sci. U. S. A., 2009, 106, 10901-10906.

3 A. Dan Peretz and D. Benedetto, Eng. Fract. Mech., 1972, 4, 979-990.

4 D. A. Dillard, B. Mukherjee, P. Karnal, R. C. Batra and J. Frechette, Soft Matter, 2018, 14, 3669-3683.

5 M. Kothari, Z. S. Lemon, C. Roth and T. Cohen, Soft Matter, 2020, 16, 9838-9843.

6 C. S. Davis, D. Martina, C. Creton, A. Lindner and A. J. Crosby, Langmuir, 2012, 28, 14899-14908.

7 W. Federle and D. Labonte, Philos. Trans. R. Soc., B, 2019, 374, 20190199.

8 A. Ghatak, Phys. Rev. E: Stat., Nonlinear, Soft Matter Phys., 2010, 81, 021603.

9 A. Majumder, A. Ghatak and A. Sharma, Science, 2007, 318, 258-261.

10 D. H. Kaelble, Trans. Soc. Rheol., 1960, 4, 45-73.

11 D. Maugis and M. Barquins, J. Phys. D: Appl. Phys., 1978, 11, 1989-2023.

12 K. Kendall, J. Phys. D: Appl. Phys., 1975, 8, 1449-1452.

13 C. Creton and M. Ciccotti, Rep. Prog. Phys., 2016, 79, 046601.

14 W. G. Bae, D. Kim, M. K. Kwak, L. Ha, S. M. Kang and K. Y. Suh, Adv. Healthcare Mater., 2013, 2, 109-113. 
15 M. K. Kwak, H. E. Jeong and K. Y. Suh, Adv. Mater., 2011, 23, 3949-3953.

16 M. D. Bartlett, E. J. Markvicka and C. Majidi, Adv. Funct. Mater., 2016, 26, 8496-8504.

17 S. Kim, J. Wu, A. Carlson, S. H. Jin, A. Kovalsky, P. Glass, Z. Liu, N. Ahmed, S. L. Elgan, W. Chen, P. M. Ferreira, M. Sitti, Y. Huang and J. A. Rogers, Proc. Natl. Acad. Sci. U. S. A., 2010, 107, 17095-17100.

18 Y. Tang, Q. Zhang, G. Lin and J. Yin, Soft Robot., 2018, 5(5), 592-600.

19 A. B. Croll, N. Hosseini and M. D. Bartlett, Adv. Mater. Technol., 2019, 1900193.

20 A. Ghatak, L. Mahadevan, J. Chung, M. Chaudhury and V. Shenoy, Proc. R. Soc. London, Ser. A, 2004, 460, 2725.

21 M. D. Bartlett, A. B. Croll, D. R. King, B. M. Paret, D. J. Irschick and A. J. Crosby, Adv. Mater., 2012, 24, 1078-1083.

22 M. Tatari, A. Mohammadi Nasab, K. T. Turner and W. Shan, Adv. Mater. Interfaces, 2018, 5, 1800321.

23 S. J. R. Brian, T. Michal and E. J. Spencer, ACS Appl. Mater. Interfaces, 2016, 8, 11041-11049.

24 C. M. J. Krahn and D. Sameoto, Smart Mater. Struct., 2010, 20, 015014.

25 J. Eisenhaure and S. Kim, Polymers, 2014, 6, 2274-2286.

26 M. Frensemeier, J. S. Kaiser, C. P. Frick, A. S. Schneider, E. Arzt, R. S. Fertig and E. Kroner, Adv. Funct. Mater., 2015, 25, 3013-3021.

27 J. D. Eisenhaure, T. Xie, S. Varghese and S. Kim, ACS Appl. Mater. Interfaces, 2013, 5, 7714-7717.

28 S. Song, D.-M. Drotlef, C. Majidi and M. Sitti, Proc. Natl. Acad. Sci. U. S. A., 2017, 201620344.

29 V. Levering, Q. Wang, P. Shivapooja, X. Zhao and G. P. López, Adv. Healthcare Mater., 2014, 3, 1588-1596.
30 N. Nadermann, J. Ning, A. Jagota and C.-Y. Hui, Langmuir, 2010, 26, 15464-15471.

31 A. Carlson, S. Wang, P. Elvikis, P. M. Ferreira, Y. Huang and J. A. Rogers, Adv. Funct. Mater., 2012, 22, 4476-4484.

32 M. D. Swift, C. B. Haverkamp, C. J. Stabile, D. Hwang, R. H. Plaut, K. T. Turner, D. A. Dillard and M. D. Bartlett, Adv. Mater. Technol., 2020, 2000676.

33 M. Manti, V. Cacucciolo and M. Cianchetti, IEEE Robot. Autom. Mag., 2016, 23, 93-106.

34 N. G. Cheng, M. B. Lobovsky, S. J. Keating, A. M. Setapen, K. I. Gero, A. E. Hosoi and K. D. Iagnemma, Proceedings of 2012 IEEE International Conference on Robotics and Automation (ICRA), 2012, pp. 4328-4333.

35 Y. C. Ying Wei Tao Ren, Q. Chen, C. Yan, Y. Yang and Y. Li, Soft Robot., 2016, 3, 134-143.

36 A. Jiang, T. Ranzani, G. Gerboni, L. Lekstutyte, K. Althoefer, P. Dasgupta and T. Nanayakkara, Soft Robot., 2014, 1, 192-201.

37 E. Brown, N. Rodenberg, J. Amend, A. Mozeika, E. Steltz, M. R. Zakin, H. Lipson and H. M. Jaeger, Proc. Natl. Acad. Sci. U. S. A., 2010, 107, 18809-18814.

38 L. Li, Z. Liu, M. Zhou, X. Li, Y. Meng and Y. Tian, Smart Mater. Struct., 2019, 28, 115023.

39 S. Xia, L. Ponson, G. Ravichandran and K. Bhattacharya, Phys. Rev. Lett., 2012, 108, 196101.

40 D. G. Hwang, K. Trent and M. D. Bartlett, ACS Appl. Mater. Interfaces, 2018, 10, 6747-6754.

41 K. Kendall, Proc. R. Soc. A, 1975, 341, 409-428.

42 R. Garg and N. V. Datla, J. Adhes., 2019, 95, 169-186.

43 S. Xia, L. Ponson, G. Ravichandran and K. Bhattacharya, J. Mech. Phys. Solids, 2013, 61, 838-851. 